\title{
Data Visualization to Promote Understanding of the Community Health Impact of the Flint Water Crisis
}

\author{
Annika Nikhar ${ }^{1}$ and Daniel Kruger" \\ ${ }^{1}$ Winston Churchill High Schoo, Potomac, MD, USA \\ "Advisor
}

$\underline{\text { ABSTRACT }}$

Between 2014 to 2015, the city of Flint suffered from critically contaminated water that caused long-term adverse health effects for many of its residents. In 2017, the "Speak to Your Health!" Community Survey assessed various aspects of adult Flint residents' health. In addition to the health-related assessments, there were also questions about residents' tap water quality, including during the times of Flint's water crisis. This project used results from the survey on how water turbidity levels affected the number of days when poor mental and physical health interfered with daily activities. Diagramming software was used to create images representing this relationship in intuitive formats. These data visualizations were intended to boost data literacy among non-researchers, particularly the people and policymakers of Flint. Given that the crisis ensued after Flint's water source was switched without proper infrastructure in place to ensure appropriate water quality, causing the population to suffer long term health, social, and economic complications, it is hoped that these results will be used to empower the population of Flint to advocate for continued investment in remediation and prevent future similar health crises.

\section{Introduction}

In 2014, the city of Flint began using the Flint River as its primary water source, with treatment facilities at the Flint Water Service Center (FWSC). This development occurred because Flint's previous water source - treated water purchased from the Detroit Water and Sewage Department (now known as the Great Lakes Water Authority) - had continuously increased in price, and costs were soon considered too demanding. To save money and find a new water source, Flint's city council decided to join the Karegnondi Water Authority (KWA), a Michigan-based corporation responsible for distributing water across the state. However, during the construction of a KWA pipeline from Lake Huron, Flint temporarily switched its water source to the Flint River (Zahran et al., 2017). During this transition, FWSC failed to treat the highly polluted river water adequately, overlooking federally mandated anti-corrosive compounds, which caused highly corroded pipes to leach dangerous levels of lead and toxins into the water supply of households across Flint. When Michigan Attorney General Bill Schuette launched an investigation to determine the number of laws broken during the crisis, fifteen people were found criminally responsible (Denchak, 2018). For eighteen months, beginning in April of 2014 and ending in October of 2015 (Zahran et al., 2017), this debacle had evident effects on water quality and, more importantly, the health of Flint's population (Bosman, 2016).

Through this time, the people of Flint voiced their concerns to authorities, pointing out their water's heavy discoloration, odor, taste, and ensuing medical conditions, eg. skin inflammation and hair loss that had begun after the switch. Additionally, reports of harmful bacteria such as Escherichia coli and Legionella resulted in a series of alerts advising residents to boil their tap water before drinking (Basha, 2016). Testing of water for contaminants revealed significantly elevated lead levels that were later attributed to runoff from corroded pipes. Lead, a seriously toxic element, can be extremely detrimental to human health even in small doses, as it can bioaccumulate. Especially for a child's development, where lead is far more potent, it should not be tolerated in any doses and, in the United States, has a maximum contaminant goal in drinking water of zero (EPA, 2021). According to water samples collected from 
252 Flint households in 2015, over 40\% indicated lead levels above 5 ppb (parts per billion), and 17\% reported levels above 15 ppb (Edwards, M., Roy, S., Rhoads, W., 2015). Furthermore, prevalence of elevated lead levels in blood had nearly doubled in children across Flint and even tripled in select neighborhoods between 2014 and 2015 (HannaAttisha et al., 2016).

An abundance of medical ailments, including high blood pressure and heart, brain, and kidney damage, was seen in adults, and exposure to lead has been known to cause impaired learning and development in pediatric age groups. According to data from 2019, approximately $28 \%$ of students in the Flint area qualified for special education services, and 70\% required accommodations for learning disorders, such as ADHD, dyslexia, etc. - drastic increases from special education data prior to the Flint Water Crisis (Green, 2019). These outcomes not only affected the children themselves, but also parents and teachers who shared the burden of additional care. Throughout and after the crisis, many adults struggled with depression, sleeping problems, anxiety, post-traumatic stress disorder, and more. Lack of trust in the Flint government, fear, and stigma led to increases in coping strategies, often involving drugs, alcohol, and other risky behaviors (Brooks, Patel, 2021). Years after the city stopped using the Flint River as a primary water source, Flint residents continued to report additional monthly expenditures from continuous purchases of bottled water, rather than relying on their provided tap water (Basha, 2016). Not only did the Flint Water Crisis pose negative health consequences, but it also caused huge economic setbacks.

This project aims to advocate health literacy and empower the population of Flint with evidence that they can comprehend and use to enact change. Data visualization is a process of representing information through images, typically including pie charts, bar graphs, line graphs, and more. Intrinsically, data visuals appeal to the human mind with universal images, such as circles and lines, to convey patterns and trends in data (Sadiku et al., 2016). For nonresearchers, or people unfamiliar with certain datasets, data visuals can convey overall trends in an understandable manner. Such imagery makes data more accessible, allowing non-researchers to analyze and utilize data in ways that otherwise may not have been possible, thus boosting data literacy. In a time of rapid advancement, particularly in science, technology, engineering, and math (STEM), and increased opportunity for agnotology and manufactured misinformation, data literacy is an imperative skill (Gibson, J., Mourad, T., 2018). The people of Flint must understand and be cognizant of the background, causes, and effects of the Flint Water Crisis to take appropriate and reasoned decisions in the betterment of their city. Scientific data objectively conveys such information, and data literacy is necessary to utilize it.

\section{Methods}

In 2017, the "Speak to your Health!" Community Survey was conducted by a survey committee comprising community, health sector, and academic partners. Distributed to 335 adult residents of Flint, this survey asked 82 questions on varying aspects of mental, physical, and social heath to determine the relationship between increasing water turbidity and health status. The survey was distributed by postcards mailed to randomly selected specific adults in each residential Census Tract of the county included on U.S. Post Office address lists. These individuals could complete an online questionnaire on the Qualtrics survey platform, or a hard copy survey returned via a postage paid envelope upon request. Participants were also recruited via e-mails to participate in previous survey waves, postings on electronic social media, and community-based data collection events. Recruitment efforts were informed by ongoing demographic analysis of current survey participants to maximize the population representation of the survey sample.

To mitigate biases, the beginning of the survey had no obvious connection to the Flint Water Crisis, as no waterrelated questions were asked. Questions about experience with the crisis were asked towards the end of the survey, after all other health-related questions were answered. Question five of the survey began with, "In the past 30 days, for how many days did," after which two related questions were asked assessing how poor physical and mental health, respectively, kept the respondent from doing his or her usual activities, such as self-care, work, or recreation. Question seventy presented five images of varying water turbidity, as shown below. 


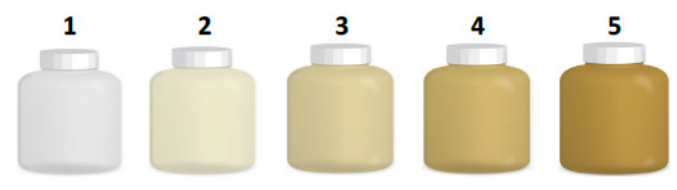

Figure 1. Tap Water Turbidity by Color

This display was paired with the question, "Which picture best represents how the tap water looked where you lived on average," for which participants would select (based on the turbidity color) what their water quality looked like in 2013, 2015, and today.

Upon conducting this survey, levels of turbidity and corresponding average days of poor mental and physical health were obtained from each participant. This information was then displayed through a series of carefully created images. For accuracy and exact measurements in these visualizations, diagramming software and spreadsheets were used in conjunction. To begin, days of poor mental and physical health were added to make clear distinctions between the days of poor health and days of good health. By doing so, the time lost to poor health could be clearly presented. Two displays, each with five images, were created to present the increasing days of poor health in relation to increasing water turbidity levels. The most basic of these displays included calendar images, in which days of poor health (lost to mental or physical challenges) were shaded in gray, while days of good health were shaded in the corresponding turbidity color. The next display involved 12-hour clocks - an obvious symbol of time - to present the increasing amount of time lost to poor mental or physical health. For each image, $x$ was used to represent the proportional time of poor mental and physical health on a 12-hour clock, and $d$ represented the average number of days of poor mental and physical health in the past 30 days. The following calculation was used to find $x$ for each level of turbidity.

$$
x=\frac{d 12}{30}
$$

Then, $x$ hours were shaded in gray on the clock to illustrate the amount of time with poor health, while time with good health was shaded in a corresponding turbidity color. To add more context and familiarity to these images, clock dials, hands, and percentage labels were included.

Apart from these large displays, two more visuals were created that represented the full correlation between increasing time of poor health (and, conversely, decreasing time of good health) and turbidity level without five separate iterations. The first one featured a similar 12-hour clock format with overlapped wedges, each in the shade of its respective turbidity level, representing the increasing time of poor health. Once again, clock dials, hands, and labels were added for familiarity and context. The second of these combined images featured as a bar graph, where the vertical axis represented 30 days, and the horizontal axis represented turbidity levels. Each bar indicated the number of days with good health in relation to a certain turbidity level, shaded in the respective turbidity color.

\section{Results}

Below are the images created. 


\section{Journal of Student Research}
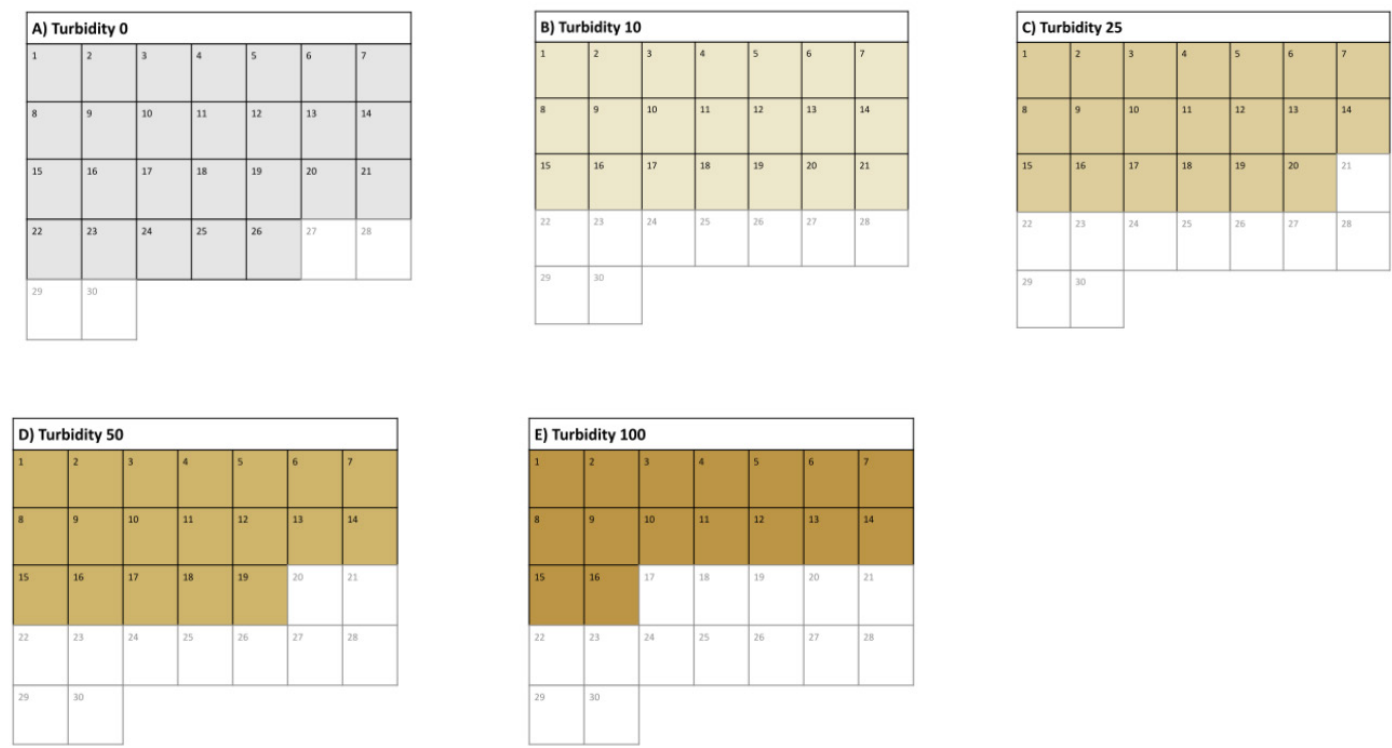

Each calendar depicts 30 days. The colored days represent days of good health and correspond to respondents' indicated turbidity level. Days of poor heath have a gray cast, demonstrating that poor mental or physical health hindered respondents from participating in their daily tasks, including self-care, work, or recreation. With increasing turbidity, days of good health shrank from twenty-six to merely sixteen. This display is perhaps the most objective, as it directly represents the data collected from the survey. 

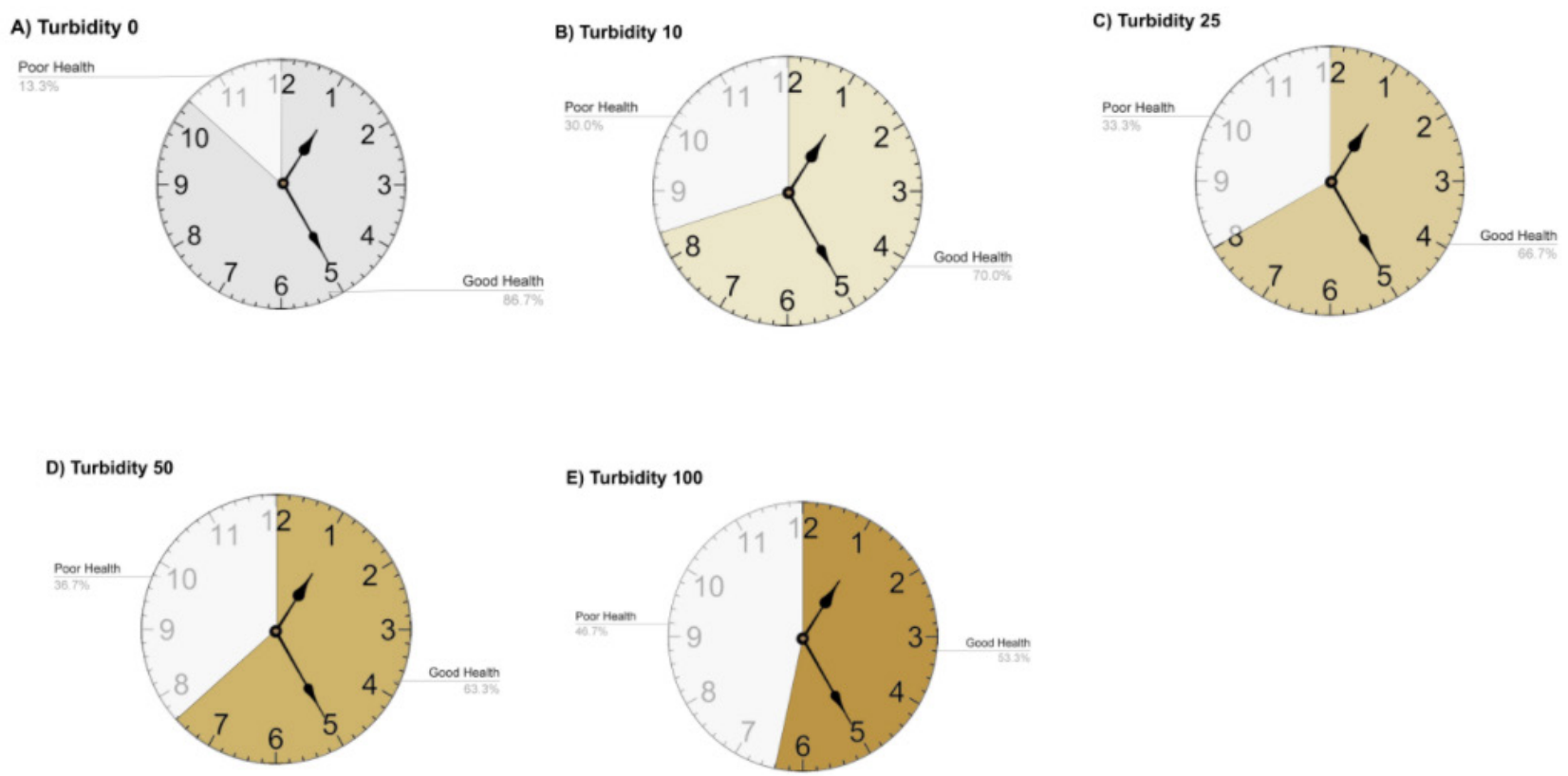

Figure 3. Clock Images Representing Time of Good Health Per Twelve Hours by Water Turbidity Levels

These images demonstrate proportional time lost on a 12-hour clock display. For each turbidity level, an average number of days of poor mental and physical health in a 30-day span was reported. This information was converted to hours of poor health in a 12-hour span. These hours were shaded in gray, while the converse time proportional hours of good health - were shaded in a corresponding turbidity color. Each image includes clock dials and hands to provide familiarity to viewers. Furthermore, exact percentages indicating proportions of poor or good health are shown with labels. By converting the time lost in a 30-day span to that in a day-to-day 12-hour span, one can clearly see the lasting impacts of poor water quality on aspects other than the health of Flint residents. Not only do these people bear negative health consequences, but their productivity and capabilities are compromised, which can have various effects to themselves, their friends, and their families. 
Figure 4. Layered Wedges Representing Time of Poor Health Per Twelve Hours by Water Turbidity Levels

This image combines information presented in the previous displays, which included five separate images for each turbidity level. In Figure 4, a single clock is shown, where wedges representing hours of poor health proportional to data conveying days of poor health in a span of 30 days (per the survey question) are overlapped. Respondents who reported water with turbidity levels of 0 , indicated by the lightest shade, typically also reported the fewest hours of poor health. Therefore, the wedge corresponding to turbidity 0 is the smallest. As time of poor health increased, the

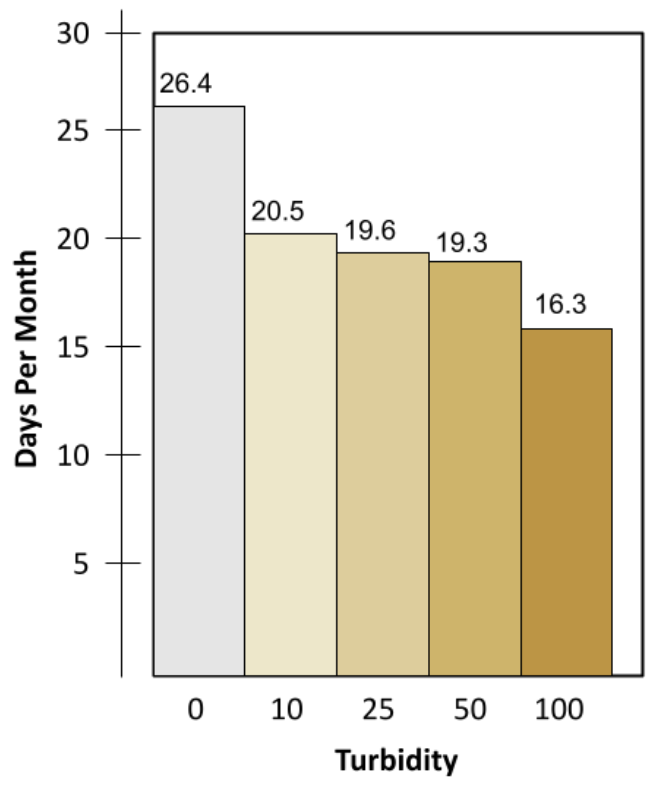

*Shaded portions indicate days of good health

Figure 5. Average Days of Good Health Per Month by Water Turbidity Levels

wedge sized increased, ultimately being the largest for turbidity 100. Excess hours were shaded in gray, time stamps were added, and clock hands were included to provide more context to the image's purpose.

Unlike Figure 4, Figure 5 depicts days of good health in relation to increasing turbidity (rather than days of poor health). This image most directly represents data collected from the survey, as the exact averages (of days of good health) are indicated by each bar. Once again, a negative trend is shown, with decreasing time of good heath for increasing turbidity levels.

\section{Discussion}


In every display, a clear correlation between water turbidity level and time of good and poor health, respectively, is shown. A positive trend between time of poor health and increasing turbidity levels was revealed, suggesting that, typically, Flint residents who were subjected to water with more contamination suffer from greater mental and physical health challenges than residents subjected to less tainted water. The converse relation is observed for time of good health, which decreased as water turbidity levels increased.

When creating these images, several iterations were made and carefully assessed to determine which were most effective in conveying data on the long-term health effects of the Flint Water Crisis. In this assessment, various community partners, including the "Speak to Your Health!" survey committee, were shown different versions, to which their feedback was taken into consideration. The overwhelming response was positive, as most partners and outside sources were enthusiastic about these new images which made data more accessible to Flint residents, even claiming that they were a "home run" in progress after the crisis. Apart from positive feedback, these visualizations were also used in a grant application to the U.S. Office of Minority Health.

Of the feedback received, it was found that the standard clock and calendar images were most intuitive and understandable. Therefore, images of these type were selected. Additionally, it was suggested that any time lost was to be shaded in gray. This suggestion allowed for total times to be shown - 30 calendar days or12-hour periods - and proportions of good or poor health to be distinctly represented. Gray portions signified that time that was inaccessible to Flint residents, due to mental or physical health constraints.

In the creation of the products, image familiarity and aesthetic were taken into consideration to create the most effective and efficient products. To make the data more accessible, we sought to add greater context to our products by using commonly known images, thus allowing interpreters to gain a sense of familiarity and build a connection. Therefore, viewers would have a baseline to begin understanding the visuals with, allowing for an easier route to comprehending the data represented. Additionally, aesthetic appearance was a heavily considered factor. Aesthetic, or the perceived beauty of the images, has correlations with task abandonment, indicating that users who find greater beauty in certain visualizations will likely have greater patience in interpreting the images as well (Cawthon, N., Moere, A., 2007). With a greater inclination to interpret the data, even finding creative ways to do so, aesthetic boosts efficiency and effectiveness. Although hard to measure aesthetic, consistent colors, fonts, and styles were used. Furthermore, context for the images, such as clock dials and hands or calendar dates, were incorporated.

To improve this project, a greater audience for feedback would have been valuable. Had there been a wider range of people, particularly Flint residents, to survey, our efficiency and product may have been improved. Ultimately, this project's purpose was to empower the people of Flint with evidence of the negative long-term health effects that they experience. Therefore, their comprehension and comfort with the created visualizations was highly valued, and greater feedback from them perhaps would have increased the efficacy of our end products. Another audience for our images included policymakers and those in legislative authority, although such feedback was received when survey partners and committee members were questioned.

We also acknowledge that recall bias was another challenge of this project. Recall bias is when information from personal history becomes skewed, typically with more significance being placed on certain events (Raphael, 1987). The "Speak to Your Health!" Community survey was conducted in 2017, approximately two years after the Flint Water Crisis ended. Although this project aimed to assess and visually represent the long-term health effects of the crisis, doing so left room for error in survey participants recollecting their experiences. Depending on how traumatic the crisis was and the number of negative effects it posed, among other factors, participants may have recalled their past water turbidity at different, presumably higher, levels.

Results from the data visualization that illustrate the correlation between past contamination in Flint drinking water and long-term adverse health effects may be used to further educate residents of Flint and legislative authorities. Future steps may include making these images more accessible, thus empowering Flint residents to advocate for improved infrastructure and greater representation in policy making. Furthermore, by increasing data literacy surrounding this topic, policy makers themselves can enact changes to mitigate risks of similar crises happening in the future. However, in this process, the purpose behind the created images must be clearly explained. Apart from solely 
distributing illustrated data, the importance and significance of the long-term health effects of the Flint Water Crisis must be understood for any advocacy or change to be enacted (Gibson, J., Mourad, T., 2018). Additionally, the purpose behind increased accessibility of such data must be known, being an essential component of the ultimate goal of these data visualizations.

\section{Conclusion}

Data from the "Speak to Your Health!" Community survey showed positive correlations between water turbidity levels and time compromised by poor mental and physical health, indicating that Flint residents with higher levels of past turbidity likely have more time with mental and physical health challenges. The graphical images created in this project illustrate this trend, visually conveying the correlation of long-term community health impacts of the Flint Water Crisis and make this relationship accessible to non-researchers. These images showing visual representation of the crisis should be easily accessible and comprehensible to the population of Flint and its policymakers to help advocate for long-term and sustainable changes to the city's infrastructure and to prevent future crises.

\section{Acknowledgments}

I would like to thank Dr. Daniel Kruger, my advisor and mentor in this project. His patience, advice, and support were of tremendous help.

\section{References}

Basha, R. (2016, October 10). One year later: The long-term effects of the Flint water crisis. The Michigan Daily. https://www.michigandaily.com/government/one-year-later-look-flint-and-where-city-now/

Bosman, J. (2016, March 23). Flint Water Crisis Inquiry Finds State Ignored Warning Signs. The New York Times. https://www.nytimes.com/2016/03/24/us/flint-water-crisis.html

Brooks, S. K., \& Patel, S. S. (2021). Psychological consequences of the Flint Water Crisis: A Scoping Review. Disaster Medicine and Public Health Preparedness, 1-11. https://doi.org/10.1017/dmp.2021.41

Cawthon, N., \& Moere, A. V. (2007). The Effect of Aesthetic on the Usability of Data Visualization. 2007 11th International Conference Information Visualization (IV '07), 637-648. https://doi.org/10.1109/IV.2007.147

Denchak, M. (2018, November 8). Flint Water Crisis: Everything You Need to Know. Natural Resources Defense Council. https://www.nrdc.org/stories/flint-water-crisis-everything-you-need-know\#sec-summary

Edwards, M., Roy, S., \& Rhoads, W. (2015, September). Lead testing results for water samples by residents. Flint Water Study. http://flintwaterstudy.org/information-for-flint-residents/results-for-citizen-testing-for-lead300-kits/

Gibson, J., \& Mourad, T. (2018). The growing importance of data literacy in life science education. American Journal of Botany, 105(12), 1953-1956. https://doi.org/10.1002/ajb2.1195

Green. E. L. (2019, November 6). Flint's Children Suffer in Class After Years of Drinking the Lead-Poisoned Water. The New York Times. https://www.nytimes.com/2019/11/06/us/politics/flint-michigan-schools.html

Gross, T. (2018, June 25). Pediatrician Who Exposed Flint Water Crisis Shares Her 'Story Of Resistance'. National Public Radio. https://www.npr.org/sections/health-shots/2018/06/25/623126968/pediatrician-who-exposedflint-water-crisis-shares-her-story-of-resistance

Hanna-Attisha, M., LaChance, J., Sadler, R. C., \& Schnepp, A. C. (2016). Elevated Blood Lead Levels in Children Associated With the Flint Drinking Water Crisis: A Spatial Analysis of Risk and Public Health Response. American Journal of Public Health, 106, 283-290. https://ajph.aphapublications.org/doi/abs/10.2105/AJPH.2015.303003 
Raphael, K. (1987). Recall Bias: A Proposal for Assessment and Control. International Journal of Epidemiology, 16(2), 167-170. https://doi.org/10.1093/ije/16.2.167

Sadiku, M. N. O., Shadare, A., Musa, S. M., \& Akujuobi, C. M. (2016). Data Visualization. International Journal of Engineering Research and Advanced Technology, 2(12). https://www.researchgate.net/profile/AdebowaleShadare/publication/311597028 DATA VISUALIZATION/links/5851945608aef7d0309f20a7/DATAVISUALIZATION.pdf

United States Environmental Protection Agency (2021). Basic Information about Lead in Drinking Water. https://www.epa.gov/ground-water-and-drinking-water/basic-information-about-lead-drinking-water

Zahran, S., McElmurry, S.P., \& Sadler, R. C. (2017). Four phases of the Flint Water Crisis: Evidence from blood lead levels in children. Environmental Research, 157, 160-172.

https://doi.org/10.1016/j.envres.2017.05.028 